\title{
Analysis of Carbapenem Susceptibility Pattern among Acinetobacter isolates in a Tertiary Care Hospital
}

\author{
K. Vishnu Preya* and R. Nepoleon
}

Sree Mookambika Institute of Medical Science, Kulasekharam, Tamilnadu, India

*Corresponding author

\begin{abstract}
A B S T R A C T
Acinetobacter baumannii has emerged as an extensively-drug-resistant pathogen implicated in healthcare associated infections (HCAIs) such as ventilator associated pneumonia, urinary tract infection, bacteremia, septicemia, secondary meningitis, wound infection etc. In the recent past carbapenems had been drugs of choice for serious infections with Acinetobacter baumannii, but carbapenem resistant strains are rapidly emerging. This study assesses the prevalence and mechanisms of resistance for Meropenem, Imipenem among Acinetobacter species isolated from a tertiary care center. Aim is to study the carbapenem susceptibility pattern among Acinetobacter isolates in a tertiary care hospital. This is a prospective study carried out in a tertiary care teaching hospital in the coastal district of Tamilnadu. A total of 1411 samples were obtained from July to December 2017 were analysed. All the Acinetobacter isolates from the clinical samples were included in this study. Bacterial isolates were identified using standard methods. Antibiotic susceptibility testing was done by Kirby Bauer disc diffusion method according to the Clinical and Laboratory Standards and Institutional guidelines. The prevalence of Acinetobacter baumannii is $6.54 \%$ and maximum number of positive samples belongs to patients above the age of 60 years with a mean age of 57.13. Majority of the culture positive isolates belong to males with the maximum number of samples were from pus. $45 \%$ of the culture positive isolates were sensitive of Meropenam with $100 \%$ resistance to imipenam. The sensitivity of Meropenam in urine, pus, sputum, other samples were $55.6 \%, 47.06,66.67 \%$ and $20 \%$ respectively. For body fluids and blood both Meropenam and imipenam were resistant to all samples.
\end{abstract}

\section{Keywords}

Acinetobacter

baumannii,

Carbapenem susceptibility pattern

Article Info

Accepted:

12 February 2019

Available Online:

10 March 2019

\section{Introduction}

Acinetobacter baumannii has emerged as an extensively-drug-resistant pathogen implicated in healthcare associated infections (HCAIs) such as ventilator associated pneumonia, urinary tract infection, bacteremia, septicemia, secondary meningitis, wound infection etc.(1) Increasing incidence of Acinetobacter species causing serious nosocomial infections in hospital intensive care units are being reported worldwide. Most frequently encountered species is Acinetobacter baumannii and it is commonly associated with infections, such as bacteremia, urinary tract infection, meningitis, skin and soft tissue infections and pneumonia with high mortality rate of $30-75 \%$ in hospitalised patients.(2) One of the most striking features of Acinetobacter baumannii is its extraordinary ability to develop resistance against major antibiotic classes.(3) In the recent past carbapenems had been drugs of choice for serious infections with 
Acinetobacter baumannii, but carbapenem resistant strains are rapidly emerging. There are several factors leading to carbapenem resistance in Acinetobacter baumannii, most important being the acquisition of carbapenem hydrolysing $\beta$-lactamases. Other mechanisms include the presence of mobile genetic elements, reduced expression of outer membrane proteins, altered affinity or expression of penicillin-binding proteins and multidrug efflux pumps.(4) Acquired resistance mechanisms can act synergistically and integrate genes encoding antibioticinactivating enzymes, efflux pumps, ribosomal binding site mutations and down regulation of porin channels on the cell membrane giving rise to multidrug-resistant (MDR) isolates.(5) Because of frequent resistance to commonly used antibiotics, carbapenems have become important for managing Acinetobacter infections. However, their effectiveness is being increasingly compromised due to enzymatic modification of antibiotic molecules especially by carbapenemases and expression of efflux pumps. Acquired carbapenemases can be either metallo-beta-lactamases (MBLs) such as VIM and IMP, or non-MBL. MBL genes are mostly detected in class integrons' structures and these integrons are detected in a high proportion of Acinetobacter isolates.(6) carbapenamases are $\beta$-lactamases, which include serine- $\beta$-lactamases (KPC, OXA, GES, etc.) and metallo $\beta$-lactamases (MBLs).

The latter require metal ion zinc for their activity, which is inhibited by metal chelators like EDTA and thiol-based compounds but not by sulbactam, tazobactam and clavulanic acid.

The genes responsible for MBL production may be chromosomal or plasmid mediated and poses a threat of horizontal transfer among other Gram-negative bacteria. (7) This study assesses the prevalent mechanisms of resistance for imipenem, meropenum among
Acinetobacter species isolated from a tertiary care center.

The main objectives of this study to study the carbapenem susceptibility pattern among Acinetobacter isolates in a tertiary care hospital.

\section{Materials and Methods}

This is a prospective study carried out in Sree Mookambika Institute of Medical Sciences kulashekaram, Kanya kumari, Tamil nadu. A total of 1411 samples were obtained from July to December 2017. All the clinical isolates of Acinetobacter from urine, pus, sputum, blood cultures, vaginal swabs, ear swab, aspirated body fluids (pleural, peritoneal, ascitic), wound swabs from OPD and IPD of all departments. All the Acinetobacter isolates from the clinical samples were included in this study. Samples were collected from all age groups.

Samples those held for more than two hours at room temperature and those without proper labelling were excluded from the study. Bacterial isolates were identified using standard methods. Antibiotic susceptibility testing was done by Kirby Bauer disc diffusion method according to the Clinical and Laboratory Standards and Institutional guidelines. Antibiotic discs (Meropenem- 10 micrograms and Imipenem - 10 micrograms) were obtained from HIMEDIA, India.

\section{Results and Discussion}

\section{Prevalence of Acinetobacter baumannii}

Total Samples analysed $=1411$. Out of the total 1411 samples analysed 611 came to be culture positive i.e. $43.3 \%$ of the total sample is culture positive. Of that 611 culture positive specimens 40 grew Acinetobacter species i.e. prevalence of Acinetobacter species in culture positive samples is $6.54 \%$. 
Table.1 Distribution of Acinetobacter among various age groups

\begin{tabular}{|l|c|c|}
\hline \multicolumn{1}{|c|}{ Age (Years) } & Number & Percentage (\%) \\
\hline Less than 1 year & 3 & 7.50 \\
\hline 1-30 years & 3 & 7.50 \\
\hline 31-60 years & 10 & 25 \\
\hline Above 60 years & 24 & 60 \\
\hline Total & $\mathbf{4 0}$ & $\mathbf{1 0 0 . 0 0}$ \\
\hline
\end{tabular}

The mean age being 57.13

Table.2 Distribution of culture positive samples based on the gender of patients

\begin{tabular}{|l|c|c|}
\hline Gender & Number & Percentage (\%) \\
\hline Male & 27 & 67.50 \\
\hline Female & 13 & 32.50 \\
\hline Total & $\mathbf{4 0}$ & $\mathbf{1 0 0 . 0 0}$ \\
\hline
\end{tabular}

Table.3 Distribution of Acinetobacter among the culture samples

\begin{tabular}{|l|c|c|}
\hline $\begin{array}{l}\text { Culture } \\
\text { samples }\end{array}$ & Number & Percentage (\%) \\
\hline Urine & 9 & 22.5 \\
\hline Blood & 2 & 5 \\
\hline Pus & 17 & 42.5 \\
\hline Sputum & 6 & 15 \\
\hline Body fluids & 1 & 2.5 \\
\hline Others & 5 & 12.5 \\
\hline Total & $\mathbf{4 0}$ & $\mathbf{1 0 0 . 0 0}$ \\
\hline
\end{tabular}

Table.4 Sensitivity and resistance pattern of Carbapenems

\begin{tabular}{|l|c|c|c|c|}
\hline \multirow{2}{*}{ Drugs } & \multicolumn{2}{|c|}{ Sensitivity } & \multicolumn{2}{c|}{ Resistance } \\
\cline { 2 - 5 } & Number & Percentage (\%) & Number & Percentage (\%) \\
\hline Meropenam & 18 & 45 & 22 & 55 \\
\hline Imipenem & 0 & 0 & 40 & 100 \\
\hline
\end{tabular}

Table.5 Sensitivity and resistance pattern of Carbapenems in urine samples

\begin{tabular}{|l|c|c|c|c|}
\hline \multirow{2}{*}{ Drugs } & \multicolumn{2}{|c|}{ Sensitivity } & \multicolumn{2}{c|}{ Resistance } \\
\cline { 2 - 5 } & Number & Percentage (\%) & Number & Percentage (\%) \\
\hline Meropenam & 5 & 55.6 & 4 & 44.4 \\
\hline Imipenem & 0 & 0 & 9 & 100 \\
\hline
\end{tabular}


Table.6 Sensitivity and resistance pattern of Carbapenems in blood samples

\begin{tabular}{|l|c|c|c|c|}
\hline \multirow{2}{*}{ Drugs } & \multicolumn{2}{|c|}{ Sensitivity } & \multicolumn{2}{c|}{ Resistance } \\
\cline { 2 - 5 } & Number & Percentage (\%) & Number & Percentage (\%) \\
\hline Meropenam & 0 & 0 & 2 & 100 \\
\hline Imipenem & 0 & 0 & 2 & 100 \\
\hline
\end{tabular}

Table.7 Sensitivity and resistance pattern of Carbapenems in pus samples

\begin{tabular}{|l|c|c|l|c|}
\hline \multirow{2}{*}{ Drugs } & \multicolumn{2}{|c|}{ Sensitivity } & \multicolumn{2}{c|}{ Resistance } \\
\cline { 2 - 5 } & Number & Percentage (\%) & Number & Percentage (\%) \\
\hline Meropenam & 8 & 47.06 & 9 & 52.94 \\
\hline Imipenem & 0 & 0 & 17 & 100 \\
\hline
\end{tabular}

Table.8 Sensitivity and resistance pattern of Carbapenems in sputum samples

\begin{tabular}{|l|c|c|c|c|}
\hline \multirow{2}{*}{ Drugs } & \multicolumn{2}{|c|}{ Sensitivity } & \multicolumn{2}{c|}{ Resistance } \\
\cline { 2 - 5 } & Number & Percentage (\%) & Number & Percentage (\%) \\
\hline Meropenam & 4 & 66.67 & 2 & 33.33 \\
\hline Imipenem & 0 & 0 & 6 & 100 \\
\hline
\end{tabular}

Table.9 Sensitivity and resistance pattern of Carbapenems in body fluids

\begin{tabular}{|l|c|c|c|c|}
\hline \multirow{2}{*}{ Drugs } & \multicolumn{2}{|c|}{ Sensitivity } & \multicolumn{2}{c|}{ Resistance } \\
\cline { 2 - 5 } & Number & Percentage (\%) & Number & Percentage (\%) \\
\hline Meropenam & 0 & 0 & 1 & 100 \\
\hline Imipenem & 0 & 0 & 1 & 100 \\
\hline
\end{tabular}

Table.10 Sensitivity and resistance pattern of Carbapenems in other samples

\begin{tabular}{|l|c|c|l|c|}
\hline \multirow{2}{*}{ Drugs } & \multicolumn{2}{|c|}{ Sensitivity } & \multicolumn{2}{c|}{ Resistance } \\
\cline { 2 - 5 } & Number & Percentage (\%) & Number & Percentage (\%) \\
\hline Meropenam & 1 & 20 & 4 & 80 \\
\hline Imipenem & 0 & 0 & 5 & 100 \\
\hline
\end{tabular}


Graph.1 Distribution of Acinetobacter among various age groups

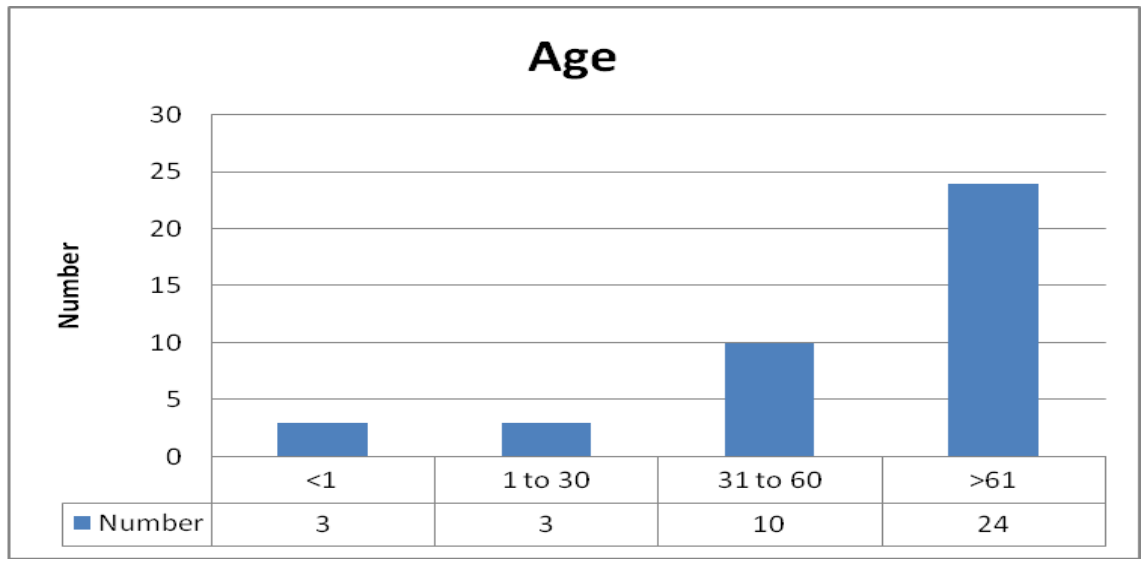

Graph.2 Gender based distribution of culture positive samples

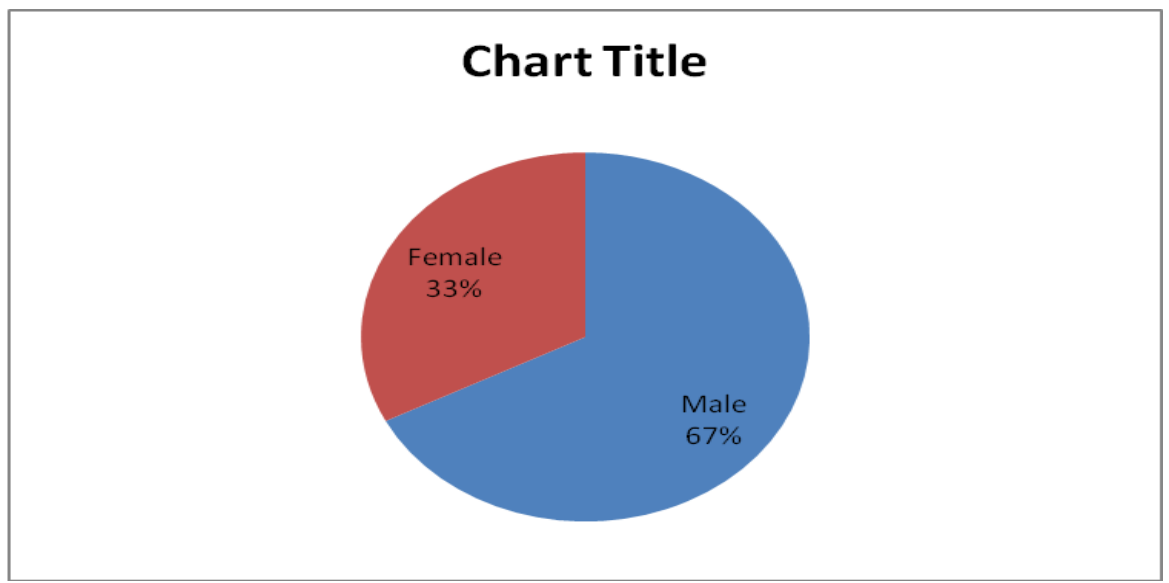

Graph.3 Distribution of Acinetobacter among the culture samples

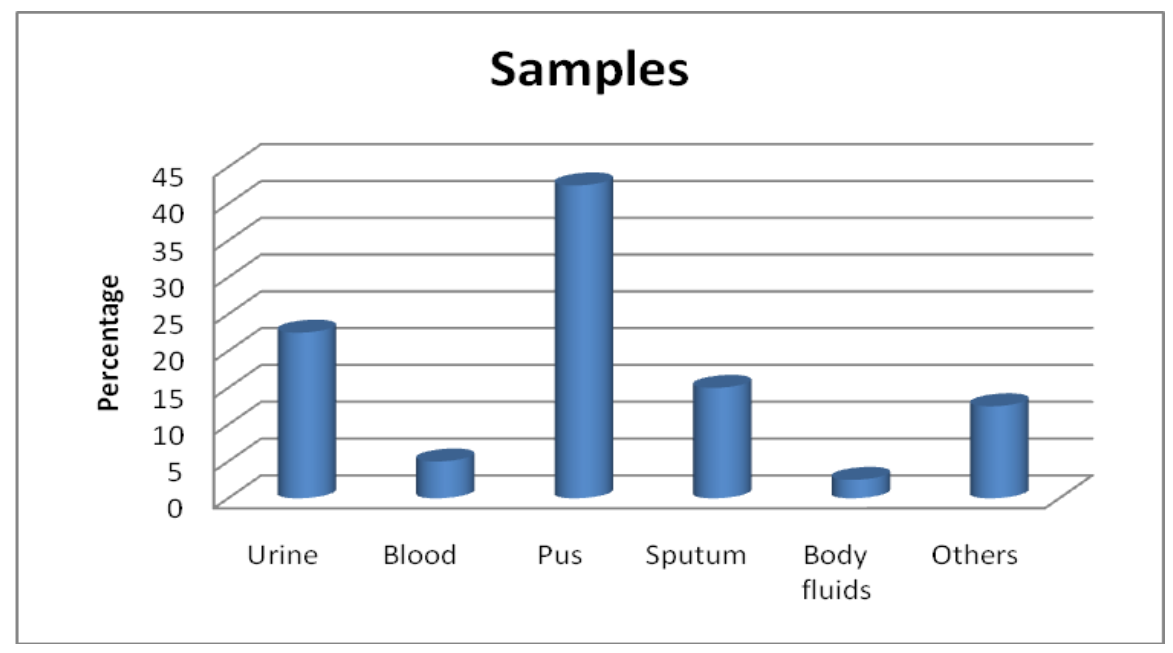




\section{Statistical analysis}

The data was expressed in number and percentage. Micro soft excel 2009 software.

Carbapenem resistance in Acinetobacter species is an emerging problem and is a cause of concern as many nosocomial Acinetobacter are detected to be resistant to most other antibiotics. Several modalities of phenotypic and molecular typing are used to detect the origin of infection, route of spread and prevalence of bacterial isolates. However, certain simple tests may be performed to determine a few common mechanisms of resistance, and these can be performed in most laboratories. In the present study, the prevalence of Acinetobacter were $6.54 \%$ among $43.3 \%$ culture positive samples when compaired with a study done by Amandeep Kaur et al., 8.8\% (48/545) Acinetobacter baumannii isolates were obtained from different samples.

In our study, $25 \%$ of samples were within the age group of 31 to 60 years. $60 \%$ of samples were above the age group of 60 years (Table 1 and Graph 1). Dr. Jhansi Charles et al., proved in a study that $80.8 \%$ were above 40 yrs. Similar study by Anuradha et al., showed more than 60 years was the common age involved. The involvement of old age group is mainly due to co morbid conditions and waning immunity which are commonly seen in the aged. Distribution of Acinetobacter infection among various isolates has been studied, which showed more predominance among males $(67.50 \%)$ than female patients (32.50\%) (Table 2 and Graph 2).

The total percentage of distribution of Acinetobacter among the culture samples showed more distribution among pus samples $(42.5 \%)$ followed by urine $(22.5 \%)$ (Table 3 and Graph 3). When compaired with a study conducted by Abhisek routray et al showed predominant distribution of Acinetobacter is among pus samples (43\%).

Amomg 40 clinical isolates of Acinetobacter, $40(100 \%)$ were found to have resistant zone sizes for Imipenem and $22(55 \%)$ showed resistance to Meropenam when tested by disk diffusion method. In our study antibiotic resistance to imipenem was $100 \%$ among clinical isolates of Acinetobacter, whereas in a study by Amarjeet Kaur, Resistance to imipenem was observed in $40.3 \%$ of $\mathrm{A}$. baumannii isolates (Table 4-10).

A study from Dr. M. Anuradha et al., Imipenem resistance was found to be $9.5 \%$. Whereas a study by Gaur A, from USA imipenem resistance was found to be $23.1 \%$. The study by Gulseran Baran et al., who showed that $53.7 \%$ were imipenem resistant in their study due to colonisation of these organisms in the devices used on these patients.

This study revealed that Imipenem resistant Acinetobacter was very common in June which is post summer and October which is post monsoon. In a study conducted by Amandeep Kaur, meropenem susceptibility is $21.8(43.8 \%)$ and $27(58.2 \%)$ were resistance. Similarly in a study of Sinha, 21 (14\%) isolates were detected to have resistant zone sizes for meropenem.

The present study demonstrates the presence of high level of multiple antibiotic resistance among carbapenem resistant Acinetobacter baumannii isolates.

A coordinated effort to limit inappropriate use of broad-spectrum antibiotics, effcient hospital antibiotic policies, vigilant detection of resistant Acinetobacters, rigorous surveillance and infection-control protocols are needed to control the increasing incidence of highly resistant Acinetobacters. 


\section{References}

1. AY, Seifert H, Paterson DL. Acinetobacter baumannii: Emergence of a successful pathogen. Clin Microbiol Rev 2008; 21: 538-82.

2. Bergogne-Bérézin E, Towner KJ. Acinetobacter spp. as nosocomial pathogens: Microbiological, clinical, and epidemiological features. Clin Microbiol Rev 1996; 9: 148-65.

3. Héritier C, Poirel L, Lambert T, Nordmann P. Contribution of acquired carbapenemhydrolyzing oxacillinases to carbapenem resistance in Acinetobacter baumannii. Antimicrob Agents Chemother 2005; 49: 3198-202.

4. Yu YS, Yang Q, Xu XW, Kong HS, Xu GY, Zhong BY. Typing and characterization of carbapenem-resistant Acinetobacter calcoaceticus- baumannii complex in a Chinese hospital. J Med Microbiol 2004; 53: 653-6.

5. Esterly J, Richardson CL, Eltoukhy NS, Qi $\mathrm{C}$, Scheetz MH. Genetic mechanisms of antimicrobial resistance of Acinetobacter baumannii (February). Ann Pharmacother 2011; 45: 218-28.

6. Seward RJ. Detection of integrons in worldwide nosocomial isolates of Acinetobacter spp. Clin Microbiol Infect 1999; 5: 308-18.

7. Varaiya A, Kulkarni N, Kulkarni M, Bhalekar P, Dogra J. Incidence of metallo$\beta$-lactamase producing Pseudomonas aeruginosa in ICU patients. Indian J Med Res 2008; 127: 398-402.

8. Kaur A, Gupta V, Chhina D. Prevalence of metalo- $\beta$-lactamase-producing (MBL)
Acinetobacter species in a tertiary care hospital. Iran J Microbiol. 2014; 6(1): 225.

9. Charles J, Rajendran, Vithiya, Ramesh. Study on the prevalence of imipenam resistant Acinetobacter species in a tertiary care hospital at Madurai. IOSR J Dent Med Sci 2017; 16(11): 37-41.

10. Anuradha, Muthulakshmi, Somasunder. Phenotypic characterization of Acinetobacter from various clinical samples and molecular study on imipenem resistant strains in a tertiary care hospital, Enathur, Kanchipuram. European J Pharmaceutical Med Res 2015;2(6):314.9

11. Gaur A, Garg A, Prakash P, Anupurba S, Mohapatra TM. Observations on carbapenem resistance by minimum inhibitory concentration in nosocomial isolates of Acinetobacter species: an experience at a tertiary care hospital in North India. J Health Popul Nutr. 2008; 26(2): 183-8.

12. Lee JY, Kang CI, Ko JH, et al., Clinical Features and Risk Factors for Development of Breakthrough Gram-Negative Bacteremia during Carbapenem Therapy. Antimicrob Agents Chemother. 2016; 60(11):6673-6678. Published 2016 Oct 21. doi:10.1128/AAC.00984-16

13. Routray A, Anu MS, Madhavan R. Colistin resistance in Acinetobacter baumannii. Int J Pharm Bio Sci 2013; 4(3):429-34

14. Kaur A, Singh S, Gill AK, Kaur N, Mahajan A. Isolation of Acinetobacter baumannii and it's antimicrobial resistance pattern in an intensive care unit (ICU) of a tertiary care hospital. Int $\mathbf{J}$ Contemp Med Res 2016; 3(6): 1794-6.

\section{How to cite this article:}

Vishnu Preya, K. and Nepoleon, R. 2019. Analysis of Carbapenem Susceptibility Pattern among Acinetobacter Isolates in a Tertiary Care Hospital. Int.J.Curr.Microbiol.App.Sci. 8(03): 1423-1429. doi: https://doi.org/10.20546/ijcmas.2019.803.166 